\title{
Intermittency of Height Fluctuations in Stationary State of The Kardar-Parisi-Zhang Equation with infinitesimal surface tension in $1+1$ Dimensions
}

\author{
S.M.A. Tabei ${ }^{a}$, A. Bahraminasab ${ }^{a}$, A. A. Masoudi ${ }^{c}$, S.S. Mousavi ${ }^{a}$ \\ and M. Reza Rahimi Tabar ${ }^{a, b}$ \\ ${ }^{a}$ Department of Physics, Sharif University of Technology, P.O. Box 11365-9161, Tehran, Iran \\ ${ }^{b}$ CNRS UMR 6529, Observatoire de la Côte d'Azur, BP 4229, 06304 Nice Cedex 4, France \\ ${ }^{c}$ Department of Physics, Alzahra University, Tehran, 19834, Iran
}

\begin{abstract}
The Kardar-Parisi-Zhang (KPZ) equation with infinitesimal surface tension, dynamically develops sharply connected valley structures within which the height derivative is not continuous. We discuss the intermittency issue in the problem of stationary state forced KPZ equation in 1+1-dimensions. It is proved that the moments of height increments $C_{a}=<\left|h\left(x_{1}\right)-h\left(x_{2}\right)\right|^{a}>$ behave as $\left|x_{1}-x_{2}\right|^{\xi_{a}}$ with $\xi_{a}=a$ for length scales $\left|x_{1}-x_{2}\right|<<\sigma$. The length scale $\sigma$ is the characteristic length of the forcing term. We have checked the analytical results by direct numerical simulation.
\end{abstract}

PACS: 05.40.-a, 68.35.Ja, 02.40.Xx, 05.45.-a

\section{INTRODUCTION}

The growth, formation and morphology of interfaces has been one of the recent interesting fields of study because of its high technical and rich theoretical advantages. On account of the disorder nature embedded in the surface growth, stochastic differential equations have been used as a suitable tool for understanding the behavior of various growth processes. Such equations typically describe the interfaces at large length scales, which means that the short length scale details has been neglected in order to derive a continuum equation by focusing on the coarse grained properties. A great deal of recent theoretical modeling has been started with the work of Edward and Wilkinson describing the dynamics of height fluctuations by a simple linear stochastic equation [1-5]. By adding a new term proportional to the square of the height gradient, Kardar, Parisi and Zhang made an appropriate description for lateral interface growth [6]. The $(1+1)$-dimensional forced KPZ equation is written as

$$
h_{t}(x, t)=\frac{\alpha}{2}\left(h_{x}\right)^{2}+\nu h_{x x}+f(x, t)
$$

where $\alpha \geq 0$ and $f(x, t)$ is a zero-mean, statistically homogeneous, white in time random force with covariance

$$
\left\langle f(x, t) f\left(x^{\prime}, t^{\prime}\right)\right\rangle=2 D_{0} D\left(x-x^{\prime}\right) \delta\left(t-t^{\prime}\right) .
$$

Typically the spatial correlation of the forcing is considered to be a delta function, mimicking the short length correlation. Here the spatial correlation is considered as

$$
D\left(x-x^{\prime}\right)=\frac{1}{\left(\pi \sigma^{2}\right)^{1 / 2}} \exp \left(-\frac{\left(x-x^{\prime}\right)^{2}}{\sigma^{2}}\right)
$$

where $\sigma$ is much less than the system size $L$, i.e. $\sigma<<L$, which represents a short range character for the random forcing. The KPZ equation has come to famous the "Ising model" of non-equilibrium physics. It is indeed the simplest equation nevertheless capturing the main determinants of the growth dynamics i.e. nonlinearity, stochasticity, and locality. The theoretical richness of the KPZ model is partly due to close relationships with other areas of statistical physics. It is shown that there is a mapping between the equilibrium statistical mechanics of a two dimensional smectic-A liquid crystal onto the non-equilibrium dynamics of the $(1+1)$ - dimensional stochastic KPZ equation [7]. It has been shown in [8] that, one can map the kinetics of the annihilation process $A+B \rightarrow 0$ with driven diffusion onto the $(1+1)$ dimensional $\mathrm{KPZ}$ equation. Also the $\mathrm{KPZ}$ equation is closely related to the dynamics of a sine-Gordon chain [9], the driven-diffusion equation $[10,11]$, high $T_{c^{-}}$superconductor [12] and directed paths in the random media [13-26] and charge density waves [27], dislocations in disordered solids [3], formation of large-scale structure in the universe [28-31], Burgers turbulence [32-60] and etc.

It is useful to rescale the $\mathrm{KPZ}$ equation as $h^{\prime}=h / h_{0}$, $\mathbf{r}^{\prime}=\mathbf{r} / \mathbf{r}_{\mathbf{0}}$ and $t^{\prime}=t / t_{0}$. If we let $h_{0}=\left(\frac{D_{0}}{\nu}\right)^{1 / 2}$ and $t_{0}=\frac{r_{0}^{2}}{\nu}$, where $r_{0}$ is a characteristic length, all of the parameters can be eliminated, except the coupling constant $g=\frac{\alpha^{2} D_{0}}{\nu^{3}}$. The limit $g \rightarrow \infty$ (or zero tension limit, $\nu \rightarrow 0)$, is known as the strong coupling limit [60]. Phase diagram information extracted from the renormalisation group flow indicates that $d=2$ plays the role of a lower critical dimension. For $d \leq 2$, the Gaussian fixed point ( $\alpha=0$ ) is infrared-unstable, and there is a crossover to the stable strong coupling fixed point. For $d>2$, a third fixed point exists, which represents the roughening transition. It is unstable and appears between the Gaussian and strong coupling fixed points which are now both stable. Only the critical indices of the strong-coupling regime $(g \rightarrow \infty$ or $\nu \rightarrow 0)$ are known in $1+1$ dimensions and their values in higher dimensions as well as properties of the roughening transition have been known only numerically [61-67], and the various approximation schemes [68-76].

For finite $\sigma$, in the strong coupling limit $(\nu \rightarrow 0)$ non- 
linear term in the KPZ equation will dominate. The nonlinearity of the KPZ equation in this limit includes the possibility of singularity formations in a finite time as a result of the local minima instability. Meaning that there is a competition between the diffusion smoothing effect ( the Laplacian term), and the enhancement of non-zero slopes. In one spatial dimension the sharp valleys are developed in a finite time. As indicated in figs.(1) and (2), the geometrical picture consists of a collection of sharp valleys intervening a series of hills in the stationary state $[77]$.

The main difficulty with the KPZ equation is that it is controlled, in all dimensions, by a strong disorder ( or strong coupling) fixed point and efficient tools are missing to calculate the exponents and other universal properties e.g. scaling functions, amplitudes, etc. Despite the fact that in one dimension, the exponents are known, but many properties, including the probability density function (PDF) of the height of a growing interface have been so far measured only in numerical simulations.

In this article, the statistical properties of the KPZ equation in the strong coupling limit $(\nu \rightarrow 0)$ is investigated. The limit is singular, i.e. through which the surface develops sharp valleys. So starting with a flat surface after a finite time scale, $t_{c} \simeq(\pi)^{1 / 6} D_{0}{ }^{-1} \alpha^{-2 / 3} \sigma^{5 / 3}[77]$, sharp valley singularities are dynamically developed. In the singular points spatial derivative of the field $h(x, t)$ is not continuous. One of the main problem in this area is the scaling behavior of moments of height increments $C_{a}=<\left|h\left(x_{1}\right)-h\left(x_{2}\right)\right|^{a}>$ and the probability density function (PDF) of $\delta h=h\left(x_{1}\right)-h\left(x_{2}\right)$, that is $P(\delta h)$. Inspired by the methods proposed recently by Weinan $\mathrm{E}$ and Vanden Eijnden [47], a statistical method is developed to describe the moments of the height and height gradient increments. We derive a master equation for joint PDF of the height and its gradient increments in $1+1$ dimensions. It is shown that in the stationary state where the sharp valleys are fully developed, the relaxation term with infinitesimal surface tension leading to an unclosed term in the PDF's equation. However we show that the unclosed term can be expressed in terms of statistics of some quantities defined on the sharp valleys. We identify each sharp valley in position $y_{0}$ with three quantities, namely the gradients of $h$ in the positions $y_{0^{+}}, y_{0^{-}}$and its height from the $\bar{h}$. The dynamics of these quantities are given in [77]. Here it is proved that to leading order, when $\left|x_{1}-x_{2}\right|<<\sigma$, fluctuation of the height field is not intermittent. The analytic form of the amplitudes of the structure functions is also given. The absence of the intermittency means that $C_{a}=<\left|h\left(x_{1}\right)-h\left(x_{2}\right)\right|^{a}>$ scales as $\left|x_{1}-x_{2}\right|^{\xi_{a}}$, where $\xi_{a}$ is a linear function of $a$. It is proved that for length scales $\left|x_{1}-x_{2}\right|<<\sigma$, the exponents $\xi_{a}$ are equal to $a$.

The paper is organized as follows; in section two, the known results for the moments of height increments $C_{a}=<\left|h\left(x_{1}\right)-h\left(x_{2}\right)\right|^{a}>$, for length scales $\left|x_{1}-x_{2}\right|>>$ $\sigma$, are expressed. In section three, we derive the master equation for the joint PDF of height and its gradient increments for given surface tension $\nu$ and for length scales $\left|x_{1}-x_{2}\right|<<\sigma$. It is shown that the surface tension term makes the PDF's equation unclosed. In section 4 we will consider the limit of $\nu \rightarrow 0$ of the master equation and derive the scaling exponents of height increments moments. Also a comparison between the analytical results and direct numerical simulation are given. Details of calculations are presented in the appendices $\mathrm{A}$ and $\mathrm{B}$.

\section{SCALING EXPONENTS OF HEIGHT-DIFFERENCE MOMENTS FOR FORCED KPZ EQUATION AND FOR THE LENGTH SCALES $\left|X_{1}-X_{2}\right|>>\sigma$.}

In this section a review of the known results for the scaling exponent of height increments moments for the KPZ equation in $1+1$-dimensions with white in time and space forcing is given. Indeed the limit $\sigma \rightarrow 0$ is considered in equation (2). In this limit the equation (2) can be written as follows;

$$
\left\langle f(x, t) f\left(x^{\prime} \cdot t^{\prime}\right)\right\rangle=2 D_{0} \delta\left(x-x^{\prime}\right) \delta\left(t-t^{\prime}\right)
$$

For this type of forcing, $\Pi[\widetilde{h}(x, t)]$, the probability functional of $\widetilde{h}(x, t)=h(x, t)-\langle h\rangle$ satisfies the functional Focker-Planck equation $[1,2]$,

$$
\begin{aligned}
\frac{\partial}{\partial t} \Pi & =\int d^{d} x \frac{\delta}{\delta \widetilde{h}(x)}\left[\left(\frac{\alpha}{2}(\nabla h)^{2}+\nu \nabla^{2} h\right) \Pi\right] \\
& +D_{0} \int d^{d} x \frac{\delta^{2}}{\delta \widetilde{h}^{2}(x)} \Pi,
\end{aligned}
$$

where its solution in the $(1+1)$-dimensions is

$$
\Pi=\exp \left[-\frac{\nu}{2 D_{0}} \int d x\left(h_{x}\right)^{2}\right] .
$$

Therefore if one introduce $G\left(x-x^{\prime}\right)=\left\langle\widetilde{h}(x) \widetilde{h}\left(x^{\prime}\right)\right\rangle$ as a Green's function, then it satisfies the following differential equation

$$
\partial_{x x} G\left(x-x^{\prime}\right)=-\frac{D_{0}}{\nu} \delta\left(x-x^{\prime}\right)
$$

so that $\langle\widetilde{h}(x) \widetilde{h}(0)\rangle=-\frac{D_{0}}{\nu}|x|$. Now we can write the second moment of height increments for small $x$ 's as

$$
\left\langle|h(x)-h(0)|^{2}\right\rangle=\frac{2 D_{0}}{\nu}|x| .
$$

In a similar way it can be seen that the higher moments, $\left\langle|h(x)-h(0)|^{a}\right\rangle$ scale with $x$ as $|x|^{\frac{a}{2}}$, which means that for the length scale $\sigma<<\left|x_{1}-x_{2}\right|<<L$, the exponents are $\xi_{a}=\frac{a}{2}$. 
There are a few comments on the result obtained for the functional PDF, equation (6). It is evident that the probability density functional (in $1+1$-dimensions ) is independent of the coefficient of the nonlinear term i.e. $\alpha$, so the result is independent of the strength of the coupling constant. As it can be seen the scaling relation is similar to an ordinary random walk problem. If one considers the random force with smooth spatial correlation, the problem changes to a more complicated one and there is no any closed solution for the functional PDF. In the next sections we will show that the moments of the height increments for the length scales $\left|x_{1}-x_{2}\right|<<\sigma$ has the scaling exponents $\xi_{a}=a$ and the amplitude of the moments are depend on the coefficient of the nonlinear term $\alpha$.

\section{THE MASTER EQUATION GOVERNING \\ THE PROBABILITY DENSITY FUNCTION OF THE HEIGHT-DIFFERENCE AND GRADIENT-DIFFERENCE FOR GIVEN SURFACE TENSION}

In this section, focusing on the $(1+1)$-dimensional KPZ equation and it's corresponding Burgers equation, the master equation describing the evolution of the joint two point PDF, $P\left(h\left(x_{1}\right)-h\left(x_{2}\right), u\left(x_{1}\right)-u\left(x_{2}\right)\right)$ of the height and corresponding height gradients increments is derived. The $(1+1)$ dimensional KPZ equation is written as

$$
h_{t}(x, t)=\frac{\alpha}{2} h_{x}^{2}+\nu h_{x x}+f(x, t)
$$

where $\alpha \geq 0$ and $f(x, t)$ is a zero-mean, statistically homogeneous, white in time random force. Its covariance is given by eq.(2). Using the map $-\partial_{x} h=u$, the corresponding Burgers equation is written as

$$
u_{t}=-\alpha u u_{x}+\nu u_{x x}-f_{x}(x, t) .
$$

Defining the two point generating function as $Z\left(\lambda_{1}, \lambda_{2}, \mu_{1}, \mu_{2}, x_{1}, x_{2}, t\right)=\langle\Theta\rangle$, where $\Theta$ is defined as,

$$
\Theta:=\exp \left(-i \lambda h_{1}-i \lambda h_{2}-i \mu_{1} u_{1}-i \mu_{2} u_{2}\right) .
$$

The fields $h_{1}$ and $h_{2}$ are the height of the surface at points $x_{1}$ and $x_{2}$. The fields $u_{1}=-\partial_{x_{1}} h_{1}$ and $u_{2}=-\partial_{x_{2}} h_{2}$ are related to the corresponding height gradients. As it is seen the generating function is the ensemble average of $\Theta$. The time evolution of $Z$ will be

$$
\begin{aligned}
Z_{t} & =-i \lambda_{1}\left\langle h_{1 t} \Theta\right\rangle-i \lambda_{2}\left\langle h_{2 t} \Theta\right\rangle \\
& -i \mu_{1}\left\langle u_{1 t} \Theta\right\rangle-i \mu_{2}\left\langle u_{2 t} \Theta\right\rangle .
\end{aligned}
$$

Using the equations (9) and (10) and noting that, in equation (12), $h_{1}, h_{2}, u_{1}$ and $u_{2}$ can be substituted by $h_{1} \rightarrow i \frac{\partial}{\partial \lambda_{1}}, h_{2} \rightarrow i \frac{\partial}{\partial \lambda_{2}}, u_{1} \rightarrow i \frac{\partial}{\partial \mu_{1}}$ and $u_{2} \rightarrow i \frac{\partial}{\partial \mu_{2}}$, the time evolution of $Z$ can be rewritten as

$$
\begin{aligned}
Z_{t} & =i \frac{\alpha \lambda_{1}}{2}\langle\Theta\rangle_{\mu_{1} \mu_{1}}+i \frac{\alpha \lambda_{2}}{2}\langle\Theta\rangle_{\mu_{2} \mu_{2}} \\
& -\alpha \mu_{1} \frac{\partial}{\partial \mu_{1}}\left\langle u_{1 x_{1}} \Theta\right\rangle-\alpha \mu_{2} \frac{\partial}{\partial \mu_{2}}\left\langle u_{2 x_{2}} \Theta\right\rangle \\
& -i \lambda_{1}\left\langle f_{1} \Theta\right\rangle-i \lambda_{2}\left\langle f_{2} \Theta\right\rangle+i \mu_{1}\left\langle f_{1 x_{1}} \Theta\right\rangle+i \mu_{2}\left\langle f_{2 x_{2}} \Theta\right\rangle \\
& +i \lambda_{1} \nu\left\langle u_{1 x_{1}} \Theta\right\rangle+i \lambda_{2} \nu\left\langle u_{2 x_{2}} \Theta\right\rangle-i \mu_{1} \nu\left\langle u_{1 x x} \Theta\right\rangle \\
& -i \mu_{2} \nu\left\langle u_{2 x x} \Theta\right\rangle .
\end{aligned}
$$

Now using ,

$$
\left\langle u_{j x} \Theta\right\rangle=\frac{i}{\mu_{j}}\langle\Theta\rangle_{x_{j}}+\frac{i \lambda_{j}}{\mu_{j}}\langle\Theta\rangle_{\mu_{j}}, \quad j=1,2
$$

the equation governing $Z$ can be written as

$$
\begin{aligned}
Z_{t} & =i \frac{\alpha \lambda_{1}}{2}\langle\Theta\rangle_{\mu_{1} \mu_{1}}+i \frac{\alpha \lambda_{2}}{2}\langle\Theta\rangle_{\mu_{2} \mu_{2}} \\
& -\alpha \mu_{1} \frac{\partial}{\partial \mu_{1}}\left(\frac{i}{\mu_{1}}\langle\Theta\rangle_{x_{1}}+\lambda_{1}\langle\Theta\rangle_{\mu_{1}}\right) \\
& -\alpha \mu_{2} \frac{\partial}{\partial \mu_{2}}\left(\frac{i}{\mu_{2}}\langle\Theta\rangle_{x_{2}}+\lambda_{2}\langle\Theta\rangle_{\mu_{2}}\right) \\
& +i \lambda_{1} \nu\left(\frac{i}{\mu_{1}}\langle\Theta\rangle_{x_{1}}+\lambda_{1}\langle\Theta\rangle_{\mu_{1}}\right) \\
& +i \lambda_{2} \nu\left\langle\frac{i}{\mu_{2}}(\Theta\rangle_{x_{2}}+\lambda_{2}\langle\Theta\rangle_{\mu_{2}}\right)+\mathcal{F}+\mathcal{G} .
\end{aligned}
$$

Here $\mathcal{F}$ and $\mathcal{G}$ stand for

$$
\begin{aligned}
\mathcal{F} & =-i \lambda_{1}\left\langle f_{1} \Theta\right\rangle-i \lambda_{2}\left\langle f_{2} \Theta\right\rangle \\
& +i \mu_{1}\left\langle f_{1 x_{1}} \Theta\right\rangle+i \mu_{2}\left\langle f_{2 x_{2}} \Theta\right\rangle \\
\mathcal{G} & =i \mu_{1} \nu\left\langle u_{1 x x} \Theta\right\rangle+i \mu_{2} \nu\left\langle u_{2 x x} \Theta\right\rangle .
\end{aligned}
$$

In equation (15) the terms $\mathcal{F}$ and $\mathcal{G}$ are the only terms which are not closed respect to $Z$. Indeed the term $\mathcal{F}$ can be also closed according to Novikov's theorem ,

$$
\begin{aligned}
\mathcal{F} & =\left(-\left(\lambda_{1}^{2}+\lambda_{2}^{2}\right) K(0)-2 \lambda_{1} \lambda_{2} K(x)\right) Z \\
& +\left(-\left(\mu_{1}^{2}+\mu_{2}^{2}\right) K(0)-2 \mu_{1} \mu_{2} K(x)\right) Z
\end{aligned}
$$

where $K(x)=2 D_{0} D(x)$ and $x=x_{1}-x_{2}$. So $G$ is the only term preventing equation (15) to be closed which can be referred to a sort of dissipative anomaly.

The PDF $P\left(h_{1}, h_{2}, u_{1}, u_{2}, x_{1}, x_{2}, t\right)$ is defined as the two-point joint probability density function (PDF) at the points $x_{1}$ and $x_{2}$ with their related heights $h_{1}$ and $h_{2}$, and their gradients $u_{1}$ and $u_{2}$. The PDF can be constructed by Fourier transforming the generating function $Z$ 
$P\left(h_{1}, h_{2}, u_{1}, u_{2}, x_{1}, x_{2}, t\right)=\int \frac{d \lambda_{1}}{2 \pi} \frac{d \lambda_{2}}{2 \pi} \frac{d \mu_{1}}{2 \pi} \frac{d \mu_{2}}{2 \pi}$

$\times \exp \left(i \lambda h_{1}+i \lambda h_{2}+i \mu_{1} u_{1}+i \mu_{2} u_{2}\right) Z\left(\lambda_{1}, \lambda_{2}, \mu_{1}, \mu_{2}, x_{1}, x_{2}\right.$

Fourier transformation of equation (15) gives the following equation for the PDF

$$
\begin{aligned}
-\frac{\partial}{\partial u_{1}} \frac{\partial}{\partial u_{2}} P_{t} & =-\frac{\alpha}{2} \frac{\partial}{\partial h_{1}} \frac{\partial}{\partial u_{1}} \frac{\partial}{\partial u_{2}}\left(u_{1}^{2} P\right) \\
& -\frac{\alpha}{2} \frac{\partial}{\partial h_{2}} \frac{\partial}{\partial u_{1}} \frac{\partial}{\partial u_{2}}\left(u_{2}^{2} P\right) \\
& -\alpha \frac{\partial}{\partial u_{2}} P_{x}+\alpha \frac{\partial}{\partial u_{1}} P_{x} \\
& -\alpha \frac{\partial}{\partial h_{1}} \frac{\partial}{\partial u_{2}}\left(u_{1} P\right) \\
& -\alpha \frac{\partial}{\partial h_{2}} \frac{\partial}{\partial u_{1}}\left(u_{2} P\right) \\
& -\alpha \frac{\partial}{\partial u_{1}} \frac{\partial}{\partial u_{2}}\left(u_{1} P_{x}\right) \\
& +\alpha \frac{\partial}{\partial u_{1}} \frac{\partial}{\partial u_{2}}\left(u_{2} P_{x}\right) \\
& +\mathcal{L}\left(\mu_{1} \mu_{2} \mathcal{F}\right)+\mathcal{L}\left(\mu_{1} \mu_{2} \mathcal{G}\right)
\end{aligned}
$$

where $x=x_{2}-x_{1}, y=\frac{x_{1}+x_{2}}{2}, \partial_{x_{1}}=-\partial_{x}+\frac{1}{2} \partial_{y}$ and $\partial_{x_{2}}=\partial_{x}+\frac{1}{2} \partial_{y}$. The terms $\stackrel{2}{\mathcal{L}}\left(\mu_{1} \mu_{2} \mathcal{F}\right)$ and $\mathcal{L}\left(\mu_{1} \mu_{2} \mathcal{G}\right)$ are the Fourier transformations of equations (16) and (17), multiplied in $\mu_{1}$ and $\mu_{2}$, where for $\mathcal{L}\left(\mu_{1} \mu_{2} \mathcal{F}\right)$ is

$$
\begin{aligned}
\mathcal{L}\left(\mu_{1} \mu_{2} \mathcal{F}\right) & =-k(0) \frac{\partial}{\partial u_{1}} \frac{\partial}{\partial u_{2}}\left(\frac{\partial^{2}}{\partial h_{1}^{2}}+\frac{\partial^{2}}{\partial h_{2}^{2}}\right) P \\
& -2 k(x) \frac{\partial}{\partial u_{1}} \frac{\partial}{\partial u_{2}} \frac{\partial}{\partial h_{1}} \frac{\partial}{\partial h_{2}} P \\
& -k_{x x}(0) \frac{\partial}{\partial u_{1}} \frac{\partial}{\partial u_{2}}\left(\frac{\partial^{2}}{\partial u_{1}^{2}}+\frac{\partial^{2}}{\partial u_{2}^{2}}\right) P \\
& -2 k_{x x}(x) \frac{\partial^{2}}{\partial u_{1}^{2}} \frac{\partial^{2}}{\partial u_{2}^{2}} P
\end{aligned}
$$

and $\mathcal{L}\left(\mu_{1} \mu_{2} \mathcal{G}\right)$ is defined as

$$
\begin{aligned}
\mathcal{L}\left(\mu_{1} \mu_{2} \mathcal{G}\right): & =-\nu\left\{\left\langle u_{1 x_{1} x_{1}} \mid h_{1}, h_{2}, u_{1}, u_{2}, x\right\rangle P\right\}_{u_{1} u_{1} u_{2}} \\
& -\nu\left\{\left\langle u_{2 x_{2} x_{2}} \mid h_{1}, h_{2}, u_{1}, u_{2}, x\right\rangle P\right\}_{u_{1} u_{2} u_{2}} .
\end{aligned}
$$

For later use we define $G$ as

$$
G:=G\left(h_{1}, h_{2}, u_{1}, u_{2}, x, t\right)+G\left(h_{1}, h_{2}, u_{1}, u_{2},-x, t\right)
$$

where

$G\left(h_{1}, u_{1}, u_{1}, u_{2}, x, t\right)=-\nu\left\{\left\langle u_{1 x_{1} x_{1}} \mid h_{1}, h_{2}, u_{1}, u_{2}, x\right\rangle P\right\}_{u_{1}}$

Also we can simply substitute $\mathcal{L}\left(\mu_{1} \mu_{2} \mathcal{G}\right)$ with $G_{u_{1} u_{2}}$. In eq. $(20), \quad\left\langle u_{1 x_{1} x_{1}} \mid h_{1}, h_{2}, u_{1}, u_{2}, x\right\rangle$ and $\left\langle u_{2 x_{2} x_{2}} \mid h_{1}, h_{2}, u_{1}, u_{2}, x\right\rangle$ are the averages of $u_{1 x_{1} x_{1}}$ and $u_{2 x_{2} x_{2}}$ conditional that the heights and velocities fields be $t) h_{1}, h_{2}, u_{1}$ and $u_{2}$ with a spatial difference $x$. Now we are interested in writing an evolution equation for the $\mathrm{PDF}^{6} \mathrm{~s}$ of height and its gradients difference. We change the variables $h_{1}, h_{2}, u_{1}$ and $u_{2}$ with $u_{1}=u-\frac{\omega}{2}, u_{2}=u+\frac{\omega}{2}$, $h_{1}=h-\frac{\xi}{2}$ and $h_{2}=h+\frac{\xi}{2}$. Integrating over $u$ and $h$ the PDF of the height and height gradient difference is obtained

$$
\begin{aligned}
& P^{\delta}(\xi, \omega, x, t) \\
= & \int d h d u P\left(h-\frac{\xi}{2}, h+\frac{\xi}{2}, u-\frac{\omega}{2}, u+\frac{\omega}{2}, x, t\right)
\end{aligned}
$$

Finally using the eq.(18), the master equation can be written as,

$$
\begin{aligned}
P_{\omega \omega t}^{\delta} & =-2 \alpha P_{x \omega}^{\delta}-\alpha\left(\omega P_{x}^{\delta}\right)_{\omega \omega}+2(k(0)-k(x)) P_{\omega \omega \xi \xi}^{\delta} \\
& +2\left(k_{x x}(0)-k_{x x}(x)\right) P_{\omega \omega \omega \omega}^{\delta}+G_{\omega \omega}^{\delta}
\end{aligned}
$$

where by considering the definition of $G$ in $(21), G^{\delta}$ would be

$$
G^{\delta}(\xi, \omega, x, t)=\int d h d u G
$$

It is clear that the $G^{\delta}$, which is proportional to surface tension $\nu$, makes the master equation unclosed. In appendix A, it is proved that for finite $\sigma$ in the limit of $\nu \rightarrow 0, G^{\delta}$ can be written in terms of the quantities which are defined on singularities.

\section{CALCULATION OF THE MOMENTS AND NUMERICAL SIMULATION}

As shown in the previous section, the presence of surface tension $\nu$, makes the master equation unclosed. However in the limit $\nu \rightarrow 0$, that is the KPZ equation with an infinitesimal surface tension, one can find exact scaling exponents of the moments \langle|$h\left(x_{1}\right)-$ $\left.\left.h\left(x_{2}\right)\right|^{a}\left|u\left(x_{1}\right)-u\left(x_{2}\right)\right|^{b}\right\rangle$. It should be noted that the $u$-field satisfying the Burgers equation, for finite $\sigma$ 's, develops discontinues or shock solutions in the limit $\nu \rightarrow 0$. Consequently for finite $\sigma$ the height field shows up as a set of sharp valleys at the positions where the shocks are located, continuously connected by some hill configurations, as indicated in Figs.(1) and (2). As mentioned, each sharp valley in position $y_{0}$ is identified by three quantities, namely the gradients of $h$ in positions $y_{0^{+}}$, $y_{0^{-}}$and it's height from $\bar{h}$. It is evident that the term $\lim _{\nu \rightarrow 0} \nu u_{x x}$ is zero at the positions where no sharp valley exists. Therefore in the limit $\nu \rightarrow 0$, only small intervals around the sharp valleys will contribute to the integral in the eq.(24). Within these intervals, boundary layer 

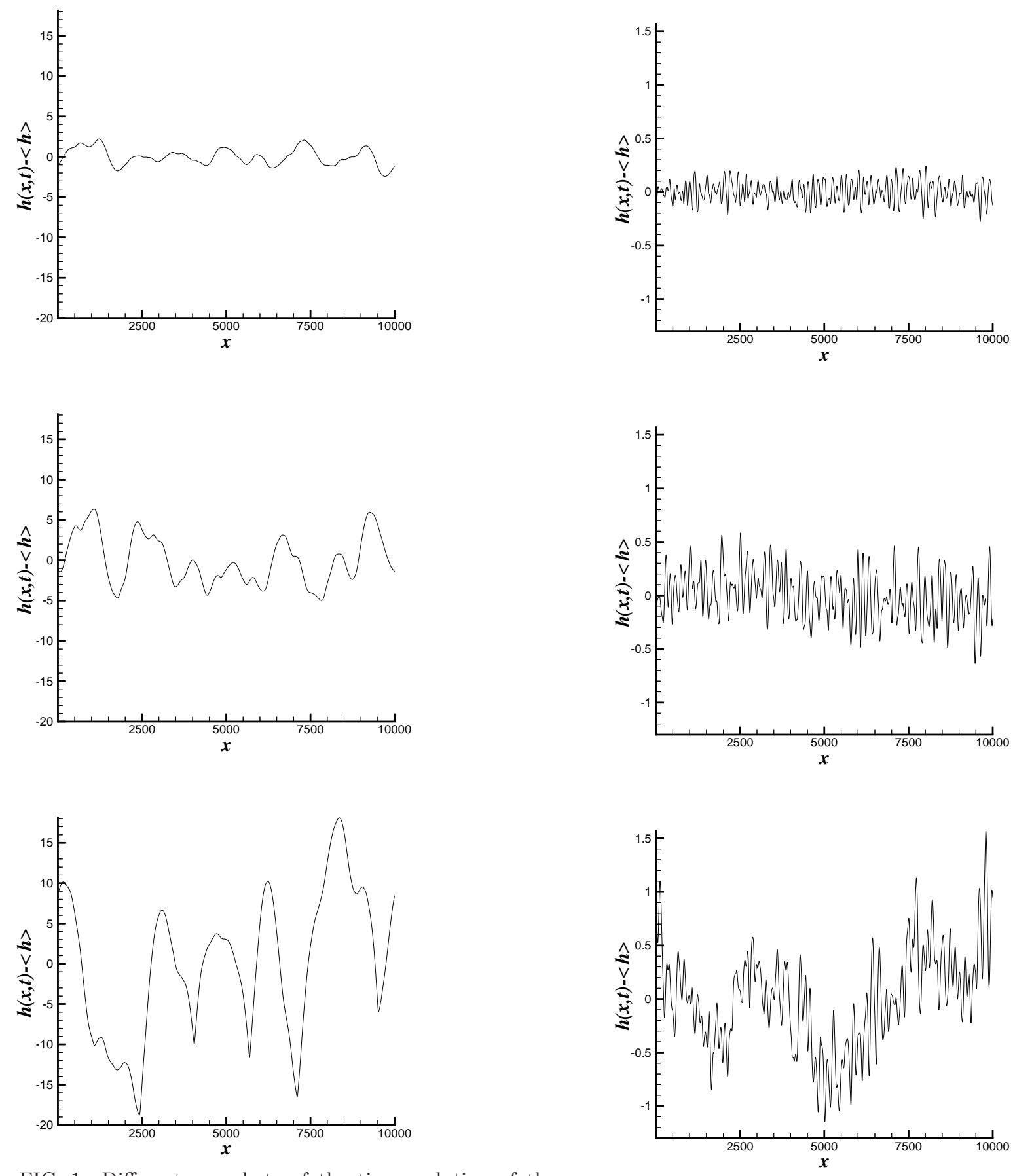

FIG. 1. Different snapshots of the time-evolution of the height, with correlation lengths $\sigma \sim L / 10$ for the random periodic force, until the time that the system finally reaches to it's stationary state. The average distance between the sharp valleys is of order of $\sigma[56,57]$.

FIG. 2. Different snapshots of the time-evolution of the height, with correlation lengths $\sigma \sim L / 100$ for the random periodic force, until the time that the system finally reaches to it's stationary state. 

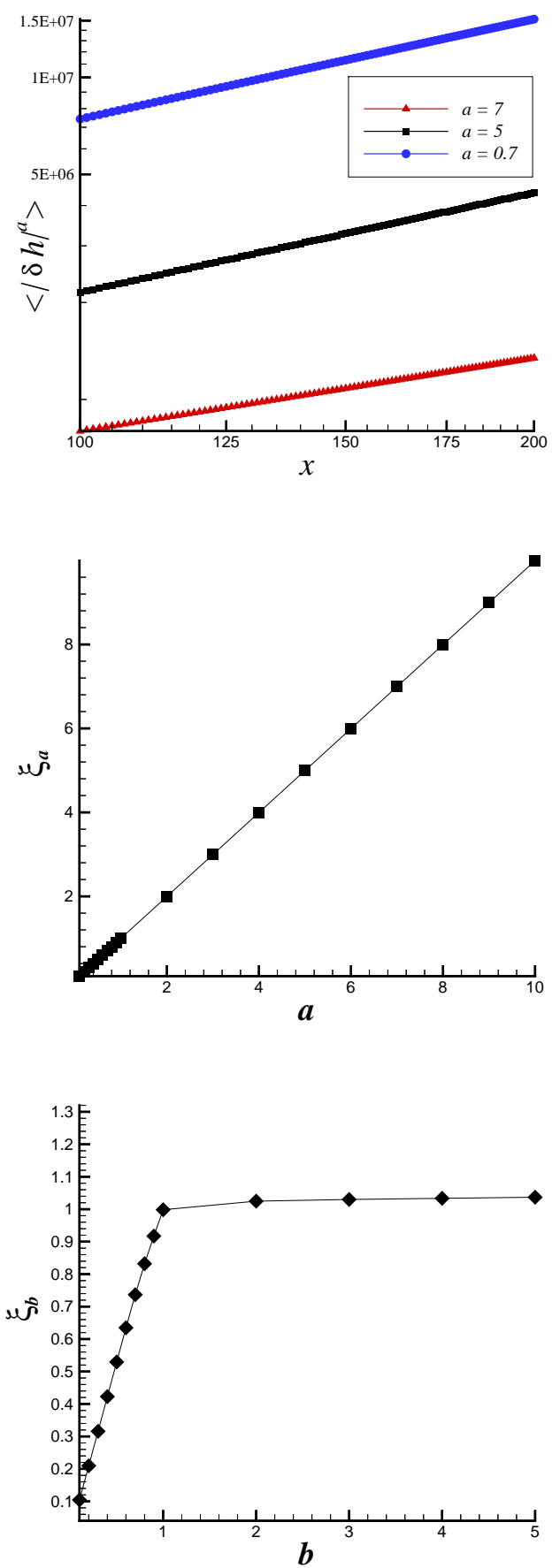

FIG. 3. In the upper graph the log-log plot of $<|h(0)-h(x)|^{a}>$ vs $x$, for moments $a=0.7,5$ and 7 are demonstrated. In the middle figure the corresponding scaling exponent $\xi_{a}$ for height increments are plotted. The $\xi_{a}$ has a linear dependence on $a$. In the lower figure the scaling exponent $\xi_{b}$ for the moments of the height gradients increments are shown. analysis can be used for obtaining an accurate approximation of $u(x, t), \tilde{h}(x, t)=h-\bar{h}$. Generally, boundary layer analysis deals with those problems in which perturbations are operative over very narrow regions where the dependent variables undergo very rapid changes across them. These narrow regions (sharp valley layers) frequently adjoin the boundaries of the domain of interest, owing the fact that a small parameter $(\nu$ in the present problem) multiplies the highest derivative. A powerful method for treating the boundary layer problems is the method of matched asymptotic expansions. The basic idea underlying this method is that an approximate solution to a given problem is sought not as a single expansion in terms of a single scale, but as two or more separate expansions in terms of two or more scales each of which is valid in part of the domain. The scales are chosen, so that the expansion as a whole, covers the whole domain of interest and the domains of validity of neighboring expansions overlap. In order to handle the rapid variations in the sharp valley layers, a suitable magnified or stretched scale and expand the functions in terms of it in the sharp valley regions is defined. For this purpose, we split $u$ and $\tilde{h}$ into a sum of inner solution near the sharp valleys and an outer solution away from the sharp valleys, and use systematic matched asymptotics to construct uniform approximation of $u$ and $\tilde{h}$. It should be emphasized that at point $y_{0}$ the height itself is continuous and height gradient (corresponding Burgers velocity) is not continuous. At these singular points the meaning of $u_{ \pm}$is that $u_{ \pm}\left(y_{0}, t\right)=u\left(y_{0 \pm}, t\right)$. Keeping in mind that $u_{-}>u_{+}$, the shock strength $s$ and the shock velocity $\bar{u}$ are defined as $s=u_{+}-u_{-}$and $\bar{u}=\frac{1}{2}\left(u_{+}+u_{-}\right)$.

In appendices $\mathrm{A}$ and $\mathrm{B}$, using the boundary layer method and the master equation, we have proved analytically, that the joint moments of the height and the corresponding gradient difference for any $a \geq 0$ will be

$$
\begin{aligned}
& \left\langle|\delta h|^{a}|\delta u|^{b}\right\rangle \\
= & \left\{\begin{array}{ll}
|x|^{a+b}\left\langle\left|\eta_{h}\right|^{a}\left|\eta_{u}\right|^{b}\right\rangle & \text { if } 0 \leq b<1 \\
|x|^{a+1}\left(\left\langle\left|\eta_{h}\right|^{a}\left|\eta_{u}\right|\right\rangle+\frac{1}{2} \rho\left\langle|s|\left(\left|u_{+}\right|^{a}+\left|u_{-}\right|^{a}\right)\right\rangle\right) & \text { if } b=1 \\
|x|^{a+1} \frac{1}{2} \rho\left\langle|s|^{b}\left(\left|u_{+}\right|^{a}+\left|u_{-}\right|^{a}\right)\right\rangle & \text { if } 1<b
\end{array}\right\}
\end{aligned}
$$

where $\delta h=h\left(x_{1}\right)-h\left(x_{2}\right), \delta u=u\left(x_{1}\right)-u\left(x_{2}\right)$ and $x=x_{1}-x_{2}$. The quantities $\eta_{h}$ and $\eta_{u}$ are the regular parts of $\partial_{x} h$ and $\partial_{x} u$, respectively. For $a=0$, our result will recover the known results for Burgers equation with infinitesimal viscosity [47].

To prove the eq.(25), we have used the fact that the length scale $\sigma$ is finite and $x$ is let to approach zero. This means that we are dealing with the scaling behaviour of the moments $\left\langle|\delta h|^{a}|\delta u|^{b}\right\rangle$ for length scales $\left|x_{1}-x_{2}\right|<<$ $\sigma$. It is evident that in these length scales the height increments fluctuations are not intermittent. Indeed we find $\xi_{a}=a$ for any moments. 
The moments of the height and height-gradient increments i.e. $\left\langle\left|h\left(x_{1}\right)-h\left(x_{2}\right)\right|^{a}\right\rangle$ and $\left\langle\left|u\left(x_{1}\right)-u\left(x_{2}\right)\right|^{b}\right\rangle$ are also calculated numerically as a function of $|x|=\left|x_{1}-x_{2}\right|$ for different $a$ 's and $b$ 's. To simulate the problem, the $\mathrm{KPZ}$ equation is discretized in space and time with scales $\delta$ and $d t$ respectively. The time scale $d t$ is related to $\delta$ as $d t=\frac{3}{4} \frac{\delta}{u_{m}}$, where $u_{m}$ is the maximum of the height gradient in each time step [78,79]. At each time step the difference $U_{j}=\left|u\left(x_{j}+1\right)-u\left(x_{j}\right)\right|$ is checked for every point $j$ 's. For $U_{j}$ 's that $U_{j}>|\delta|^{\frac{1}{3}}$, we can determine the positions that the height field develop a sharp valley [57]. Indeed this is a criterion for creation of a sharp valley in position $x_{j}=y_{j}$. At points that $U_{j}<|\delta|^{\frac{1}{3}}$ the fields $u\left(x_{j}\right)$ and $h\left(x_{j}\right)$ belong to the smooth part. Therefore the height fields $h\left(x_{j}\right)$ will fall into two regimes, points far from the sharp valleys points $y_{j}$ and the points in it's neighborhoods. For the points which the height field is regular or smooth, the height fields and it's corresponding gradients evolve under the KPZ and Burgers equation by setting the surface tension zero. Otherwise it is in the singularity or sharp valley region. As mentioned in the introduction, every sharp valley can be characterized by four parameters $s, \bar{u}, y_{j}$ and $h\left(y_{j}\right)$. The time evolution of these quantities are given by the following equations [77]

$$
\begin{aligned}
& \frac{d y_{j}}{d t}=\alpha \bar{u} \\
& \frac{d}{d t} u\left(y_{j}\right)=\frac{\alpha}{4} s\left(h_{+x x}-h_{-x x}\right)-f_{x} \\
& \frac{d}{d t} s\left(y_{j}\right)=\frac{\alpha}{2} s\left(h_{+x x}+h_{-x x}\right) \\
& \frac{d}{d t} \tilde{h}\left(y_{j}, t\right)=-\frac{\alpha}{8}\left(4 \bar{u}^{2}-s^{2}\right)+f-\gamma
\end{aligned}
$$

where $\gamma=\bar{h}_{t}$.

To calculate numerically the scaling behaviour of moments with $x$ when $x<<\sigma$, a periodic one dimensional substrate consisting a discrete $\mathrm{N}$-point height field with the length of 10000 is used. Starting with a flat initial condition the height and its gradient fields evolve in time. We consider the random force as a white in time, smooth and periodic in space random function which it's spatial correlation length is of the order of period of the given periodic function. To generate this type of forcing we use the kicking method which recently has been used in [51], to simulate the Burgers turbulence. The basic idea is that the random force can be decomposed as follows,

$$
f(x, t)=\sum_{j} f_{j}(x) \delta\left(t-t_{j}\right)
$$

where $\delta$ is the Dirac distribution and where both the "impulses" $f_{j}(x)$ and the "kicking times" $t_{j}$ are prescribed (deterministic or random). The kicking times are ordered and form a finite or infinite sequence. In this article the impulses are always taken smooth and acting only at length scales $\sigma$. Newman \& McKane [80] have used similar kicking, in a context where the forced Burgers equation is used for the study of directed polymers. Kraichnan [48] has considered a simple model in which there are non-smooth impulses creating directly saw-tooth profiles in the velocity in Burgers turbulence. Here the time intervals are equal to the time steps of the algorithm's run.

In Figs.(1) and (2), we illustrate different snapshots of the time-evolution of the height, considering different correlation lengths $\sigma$ for the random periodic force, until the time that the system finally reaches to it's stationary state. The following type of kicking force is used

$$
F(x, t)=A(t)\left[\cos (k x-\varphi(t))+\frac{1}{3} \sin (k x-\varphi(t))\right],
$$

where $A$ is a white Gaussian random variable in time, which is the noise amplitude and $\varphi$ is a homogeneous random phase. Choosing different values for $k$, leads to different values for $\sigma$. The length scale $\sigma$ is of the order of the period of $F$. In Fig.(3), the log-log plot of the moments of height increments are sketched numerically for $a=0.7,5$ and 7 , respectively. We have found the exponents $\xi_{a}=r a+q$, where $r=1.00 \pm 0.01$ and $q=$ $-0.0012 \pm 0.0002$. The scaling behaviour of the moments of height gradients increments for length scales $x<<\sigma$ is also checked. The results implies that with a good precision $\left\langle|\delta u|^{b}\right\rangle$ scales with $x$ with exponent 1 for $b$ 's larger than one, and scales with $x$ with exponents $\xi_{b}=$ $b$, for $b$ 's smaller than one with precision \pm 0.001 . The behaviour of $\xi_{b}$ vs $b$ is also plotted in fig.(3).

In summary, we study the problem of non-equilibrium surface growth described by the forced KPZ equation in $1+1$ dimensions. The forcing is a white in time Gaussian noise but with a Gaussian correlation in space with variance $\sigma$. Modeling a short range correlated noise, we restrict our study to the case when the correlation length of the forcing is much smaller than the system size. Investigating the stationary state, a general expression of the mixed correlations of height-difference and heightgradient difference at any order, in terms of the length scale $\left|x_{1}-x_{2}\right|$ and quantities which characterize the sharp valley singular structures is given. Through a careful analysis being done over the behaviour of the sharp valley environment, we decipher the intermittency exponent of an arbitrary $a$-th moment, i.e. $\left\langle\left|h\left(x_{1}\right)-h\left(x_{2}\right)\right|^{a}\right\rangle$. It is proved that the height increments fluctuations are not intermittent and its $a$-th moments for length scales $\left|x_{1}-x_{2}\right|<\sigma$ scales as $\left|x_{1}-x_{2}\right|^{\xi_{a}}$, where $\xi_{a}=a$. In the present paper the limiting of $\nu \rightarrow 0$ is taken into account only for finite $\sigma$. Still the forcing correlation length is much smaller than the system size and height correlation length. But the limit $\sigma \rightarrow 0$ is a singular limit in our calculations, and moreover, it is not a priori clear that the limits of $\nu \rightarrow 0$ and $\sigma \rightarrow 0$ commute at all. Us- 
ing stochastic equations which are governed over the dynamics of quantities characterizing the sharp valleys we simulate directly the problem and check the exponents. We have generate the forcing using the kicking method. Our simulation confirm the analytical results. We believe that the analysis followed in this paper is quite suitable for the zero temperature limit in the problem of directed polymer in the random potential with short range correlations [81]. The same method applied to KPZ equation in higher dimensions would be definitely one of the consequent goals of the present work. The main message which might be encoded in the present work is the importance of the statistical properties of the geometrical singular structures for understanding the strong coupling regime of Kardar-Parisi-Zhang equation.

\section{Acknowledgement}

We thank A. Aghamohammadi F. Azami, M. Fazeli, F. Ghasemi and F. Shahbazi for their useful discussions.

\section{Appendix A}

In this appendix we are going to prove that the $G$-term in eq.(24), has a finite value in the limit $\nu \rightarrow 0$. As shown in section 2 the $G$-term can be written as

$G=G\left(h_{1}, u_{1}, h_{2}, u_{2}, x, t\right)+G\left(h_{2}, u_{2}, h_{1}, u_{1},-x, t\right)$.

Here we prove that in the vanishing surface tension limit, the $G$ term can be written as

$G\left(h_{1}, u_{1}, h_{2}, u_{2}, x, t\right)=$

$\rho\left(\int_{-\infty}^{0} d s s \int_{u_{1}+s / 2}^{u_{1}-s / 2} d \bar{u}\left(u_{1}-\bar{u}\right) T\left(\bar{h}_{1}, \bar{u}, s, h_{2}, u_{2}, x, t\right)\right)_{u_{1}}$

where $T\left(\bar{h}_{1}, \bar{u}, s, h_{2}, u_{2}, x, t\right)$ is the PDF of

$$
\left(\bar{h}_{1}, \bar{u}\left(y_{0}, t\right), s\left(y_{0}, t\right), h_{2}\left(y_{0}+x, t\right), u_{2}\left(y_{0}+x, t\right)\right)
$$

conditional on $y_{0}$ being a sharp valley position.

Let us now prove the eq.(30). Assuming spatial ergodicity, for example the average of one of the terms in $G$, which is proportional to $\nu$, can be expressed as

$$
\begin{gathered}
\nu\left\langle u_{i x_{i} x_{i}} \mid h_{1}, h_{2}, u_{1}, u_{2}, x\right\rangle P=\nu\left\langle u_{i x_{i} x_{i}}(x, t) \delta\left(u_{1}-u_{1}\left(x_{1}, t\right)\right)\right. \\
\delta\left(u_{2}-u_{2}\left(x_{2}, t\right)\right) \delta\left(h_{1}-h_{1}\left(x_{1}, t\right)\right) \delta\left(h_{2}-h_{2}\left(x_{2}, t\right)\right\rangle \\
=\nu \lim _{L \rightarrow \infty} \frac{N}{L} \frac{1}{N} \int_{-L / 2}^{L / 2} d x_{i} u_{i x_{i} x_{i}}\left(x_{i}, t\right) \delta\left(u_{i}-u_{i}\left(x_{i}, t\right)\right) \\
\delta\left(h_{i}-h_{i}\left(x_{i}, t\right)\right) .
\end{gathered}
$$

Clearly, in the limit as $\nu \rightarrow 0$ only small intervals around the sharp valleys will contribute to the integral.
In these intervals, boundary layer analysis can be used to obtain an accurate approximation of $u_{i}(x, t)$ and $h_{i}(x, t)$. The basic idea is to split $u_{i}$ and $h_{i}$ into the sum of an inner solution near the sharp valleys and an outer solution away from the singular point, and using systematic matched asymptotic to construct uniform approximation of $u_{1}$ and $h_{i}$ (for details see, e.g., [77]). For the outer solution, we look for an approximation in the form of a series in $\nu$

$$
\begin{gathered}
h_{i}=h_{i}^{\text {out }}=h_{i}^{0}+\nu h_{i}^{1}+O\left(\nu^{2}\right) \\
u_{i}=u_{i}^{\text {out }}=u_{i}^{0}+\nu u_{i}^{1}+O\left(\nu^{2}\right) .
\end{gathered}
$$

Then $u_{i}^{0}$ and $h_{i}^{0}$ satisfy

$$
\begin{aligned}
& h_{i t}^{0}-\frac{\alpha}{2}\left(\partial_{x_{i}} h_{i}^{0}\right)^{2}=f \\
& u_{i t}^{0}+\alpha u_{i}^{0} u_{i x_{i}}^{0}=-f_{x_{i}}
\end{aligned}
$$

i.e. Burgers and KPZ equations without the surface tension terms. In order to deal with the inner solution around the singularity, let $y_{i}=y_{i}(t)$ be the position of a shock, and define the stretched variable $z_{i}=\left(x_{i}-y_{i}\right) / \nu$ and let

$$
u_{i}^{i n}(x, t)=v_{i}\left(\frac{x_{i}-y_{i}}{\nu}+\delta, t\right)
$$

where $\delta$ is a perturbation of the sharp valley position to be determined later. Then, $v_{i}$ satisfies

$$
\nu v_{i t}+\alpha\left(v_{i}-\bar{u}_{i}+\nu \gamma\right) v_{i z}=v_{i z_{i} z_{i}}+\nu f
$$

where $\bar{u}_{i}=d y_{i} / d t, \gamma=d \delta / d t$ and, to $O\left(\nu^{2}\right), \nu f$ can be evaluated at $x_{i}=y_{i}$ and can thus be considered as a function of $t$ only.

We study eq.(33) by regular perturbation analysis. We (30l)ok for a solution in the form

$$
v_{i}=v_{i}^{0}+\nu v_{i}^{1}+O\left(\nu^{2}\right) .
$$

To leading order, from eq.(33) we get for $v_{i}^{0}$ the following equation

$$
\alpha\left(v_{i}^{0}-\bar{u}_{i}\right) v_{i z_{i}}^{0}=v_{i z_{i} z_{i}}^{0} .
$$

The boundary condition for this equation arises from the matching condition with $u_{i}^{\text {out }}=u_{i}^{0}+\nu u_{i}^{1}+O\left(\nu^{2}\right)$ :

$$
\lim _{z_{i} \rightarrow \pm \infty} v_{i}^{0}=\lim _{x_{i} \rightarrow y_{i}} u_{i}^{0} \equiv \bar{u}_{i} \pm \frac{s_{i}}{2}
$$

where $s_{i}=s_{i}(t)$ is the sharp valley strength. It is understood that for small $\nu$ matching takes place for small values of $\left|x_{i}-y_{i}\right|$ and large values of $\left|z_{i}\right|=\left|x_{i}-y_{i}\right| / \nu$. (31his gives

$$
v_{i}^{0}=\bar{u}_{i}-\frac{s_{i}}{2} \tanh \left(\frac{s_{i} z_{i}}{4}\right) .
$$


These results show that, to $O(\nu)$, eq.(31) can be estimated as

$$
\begin{aligned}
& \nu\left\langle u_{i x_{i} x_{i}} \mid h_{1}, h_{2}, u_{1}, u_{2}, x\right\rangle P \\
& =\nu \lim _{L \rightarrow \infty} \frac{N}{L} \frac{1}{N} \sum_{i} \int_{\Omega_{i}} d x_{i} u_{i x_{i} x_{i}}^{i n}\left(x_{i}, t\right) \delta\left(u_{i}-u_{i}^{i n}\left(x_{i}, t\right)\right) \\
& \delta\left(h_{i}-h_{i}^{i n}\left(y_{i}, t\right)\right) \\
& =\nu \lim _{L \rightarrow \infty} \frac{N}{L} \frac{1}{N} \sum_{i} \int_{-\infty}^{\infty} d z_{i} u_{i z_{i} z_{i}}^{i n} \delta\left(u_{i}-u_{i}^{i n}\left(z_{i}, t\right)\right) \\
& \delta\left(h_{i}-h_{i}^{i n}\left(y_{i}, t\right)\right) \\
& =\nu \lim _{L \rightarrow \infty} \frac{N}{L} \frac{1}{N} \sum_{i} \int_{-\infty}^{\infty} d z_{i} v_{i}^{0} i z_{i} z_{i}^{i n} \delta\left(u_{i}-v_{i}^{0}\right) \\
& \delta\left(h_{i}-h_{i}^{i n}\left(y_{i}, t\right)\right)
\end{aligned}
$$

where $\Omega_{i}$ is a layer centered at $y_{i}$ with width $\gg O(\nu)$. Going to the stretched variable $z_{i}=\left(x_{i}-y_{i}\right) / \nu$, and using the eq.(34), we have

$$
d z v_{0 z z}=d v_{0} \frac{v_{0 z z}}{v_{0 z}}=\alpha d v_{0}\left(v_{0}-\bar{u}\right)
$$

so by taking the limit as $L \rightarrow \infty$, the $z$ integral can be evaluated exactly

$$
\begin{aligned}
& \nu\left\langle u_{1 x_{1} x_{1}} \mid h_{1}, h_{2}, u_{1}, u_{2}, x\right\rangle P \\
& \left.=\alpha \rho \int d \bar{u} \int_{-\infty}^{0} d s T \widetilde{\left(h_{1}\right.}, \bar{u}_{1}, s_{1}, \widetilde{h_{2}}, u_{2}, x ; t\right) \\
& \int_{\bar{u}_{1}+s_{1} / 2}^{\bar{u}_{1}-s_{1} / 2} d v_{1}^{0}\left(v_{1}^{0}-\bar{u}_{1}\right) \delta\left(u_{1}-v_{1}^{0}\right) .
\end{aligned}
$$

Where $\left(h_{1}, \bar{u}_{1}, s_{1}, h_{2}, u_{2}, x ; t\right) \quad$ is the PDF of $\left(h_{1}\left(y_{1}, t\right), \bar{u}_{1}\left(y_{1}, t\right), s\left(y_{1}, t\right), h_{2}\left(y_{1}+x, t\right), u_{2}\left(y_{1}+x, t\right)\right)$ conditional on $y_{1}$ being a sharp valley location and the spatial difference of the heights $h_{1}$ and $h_{2}$ be $x$. Hence,

$$
\begin{aligned}
& \nu\left\langle u_{1 x_{1} x_{1}} \mid h_{1}, h_{2}, u_{1}, u_{2}, x\right\rangle P \\
& =-\alpha \rho \int_{-\infty}^{0} d s s \int_{u_{1}+s / 2}^{u_{1}-s / 2} d \bar{u}\left(u_{1}-\bar{u}\right) T\left(\bar{h}_{1}, \bar{u}, s, h_{2}, u_{2}, x, t\right) .
\end{aligned}
$$

For late use we note that the $G$-term can be written in a more convenient manner as

$$
\begin{aligned}
& G\left(h_{1}, u_{1}, h_{2}, u_{2}, x, t\right)= \\
& \frac{\rho}{2} \int_{-\infty}^{0} d s s\left(T\left(\bar{h}_{1}, u_{1}-\frac{s}{2}, s, h_{2}, u_{2}, x, t\right)\right. \\
& \left.+T\left(\bar{h}_{1}, u_{1}+\frac{s}{2}, s, h_{2}, u_{2}, x, t\right)\right) \\
& +\rho \int_{-\frac{1}{2}}^{\frac{1}{2}} d \beta \int_{-\infty}^{0} d s s T\left(\bar{h}_{1}, u_{1}+\beta s, s, h_{2}, u_{2}, x, t\right) .
\end{aligned}
$$

\section{Appendix B}

The main aim of this appendix is to calculate the mixed moments $\left\langle|\delta h|^{a}|\delta u|^{b}\right\rangle$ by the use of master equation derived in the section 2. As we will see the term $G$ has an essential role in the results being to obtain the moments. Considering in mind equations eqs.(21) and (22), $G^{\delta}$ could be written as

$$
\begin{aligned}
& G^{\delta}(\xi, \omega, x, t)= \\
& \int d h d u G\left(h-\frac{\xi}{2}, h+\frac{\xi}{2}, u-\frac{\omega}{2}, u+\frac{\omega}{2}, x, t\right) \\
& +\int d h d u G\left(h+\frac{\xi}{2}, h-\frac{\xi}{2}, u+\frac{\omega}{2}, u-\frac{\omega}{2},-x, t\right) .
\end{aligned}
$$

It is proved in appendix A that the $G$-term can be written as follows

$G\left(h_{1}, u_{1}, h_{2}, u_{2}, x, t\right)=$

$\alpha \rho\left(\int_{-\infty}^{0} d s s \int_{u_{1}+s / 2}^{u_{1}-s / 2} d \bar{u}\left(u_{1}-\bar{u}\right) T\left(\bar{h}_{1}, \bar{u}, s, h_{2}, u_{2}, x, t\right)\right)_{u_{1}}$

where $T\left(\bar{h}_{1}, \bar{u}, s, h_{2}, u_{2}, x, t\right)$ is the PDF of

$$
\left(\bar{h}_{1}, \bar{u}\left(y_{0}, t\right), s\left(y_{0}, t\right), h_{2}\left(y_{0}+x, t\right), u_{2}\left(y_{0}+x, t\right)\right)
$$

conditional on $y_{0}$ being a sharp valley position. It should be emphasized that when we say $y_{0}$ is a singular point, we mean that however the height itself is continuous at $y_{0}$ the height gradient (corresponding Burgers velocity) is not continues at these points. At these singular points the meaning of $u_{ \pm}$is that $\left.u_{ \pm}\left(y_{0}, t\right)=u( \pm x, t)\right)$ keeping in mind that $u_{-}>u_{+}$, while the singularity strength $s$ and $\bar{u}$ are defined as $s=u_{+}-u_{-}$and $\bar{u}=\frac{1}{2}\left(u_{+}+u_{-}\right)$. We define $h_{+}\left(y_{0}, t\right)$ and $h_{+}\left(y_{0}, t\right)$ as

$$
\begin{aligned}
& h_{+}\left(y_{0}, t\right)=\bar{h}\left(y_{0}\right)+\frac{\epsilon}{2} \\
& h_{-}\left(y_{0}, t\right)=\bar{h}\left(y_{0}\right)-\frac{\epsilon}{2}
\end{aligned}
$$

Due to the continuity of $h$ the limit $\epsilon \rightarrow 0$ is not singular. Now let us rewrite the $G^{\delta}$ in a manner to be more convenient for the rest of the calculations. For this purpose let

$$
\begin{aligned}
& \delta u_{+}\left(x, y_{0}, t\right)=u\left(y_{0}+|x|, t\right)-u_{+}\left(y_{0}, t\right) \\
& \delta u_{-}\left(x, y_{0}, t\right)=u_{-}\left(y_{0}, t\right)-u\left(y_{0}-|x|, t\right) \\
& \delta h_{+}\left(x, y_{0}, t\right)=h\left(y_{0}+|x|, t\right)-h_{+}\left(y_{0}, t\right) \\
& \delta h_{-}\left(x, y_{0}, t\right)=h_{-}\left(y_{0}, t\right)-h\left(y_{0}-|x|, t\right)
\end{aligned}
$$

and define $U_{ \pm}\left(\epsilon, s, \delta h_{ \pm}, \delta u_{ \pm}, x, t\right)$ be the PDF's of $\left(\epsilon, s\left(y_{0}, t\right), \delta h_{ \pm}\left(x, y_{0}, t\right), \delta u_{ \pm}\left(x, y_{0}, t\right)\right)$ conditional on $y_{0}$ 
being a sharp valley position. Then $G^{\delta}$ can be expressed as

$$
G^{\delta}(\xi, \omega, x, t)=G_{+}^{\delta}(\xi, \omega, x, t)+G_{-}^{\delta}(\xi, \omega, x, t)
$$

where

$$
\begin{aligned}
& G_{ \pm}^{\delta}(\xi, \omega, x, t)= \\
& \alpha \frac{\rho}{2} \int_{-\infty}^{0} d \operatorname{ss}\left[U_{ \pm}(\epsilon, s, \operatorname{sgn}(x) \xi-\epsilon, \operatorname{sgn}(x) \omega-s, x, t)\right. \\
& \left.+U_{ \pm}(\epsilon, s, \operatorname{sgn}(x) \xi, \operatorname{sgn}(x) \omega, x, t)\right] \\
& -\alpha \rho \int_{-\infty}^{0} d s s \int_{0}^{1} d \beta U_{ \pm}\left(\epsilon, s, \operatorname{sgn}(x) \xi-\frac{\epsilon}{2}, \operatorname{sgn}(x) \omega-\beta s, x, t\right) .
\end{aligned}
$$

We are interested in scaling behaviour of mixed moments in small length scale $x$. In the limit $x \rightarrow 0$ it should be noted that $P^{\delta}$ can be decomposed into two parts as

$$
\begin{aligned}
P^{\delta}(\xi, \omega, x, t) & =p_{n s}(x, t) P^{\delta}(\xi, \omega, x, t \mid \text { no sharp valley }) \\
& +\left(1-p_{n s}(x, t)\right) P^{\delta}(\xi, \omega, x, t \mid \text { sharp valley })
\end{aligned}
$$

where $p_{n s}(x, t)$ is the probability that there is no sharp valley in $[y, y+x)$ and $P^{\delta}(\xi, \omega, x, t \mid$ no sharp valley $)$ is the PDF of $\delta u(x, y, t)$ and $\delta h(x, y, t)$ conditional on the property that there is no sharp valley in $[y, y+x)$. Also $P^{\delta}(\xi, \omega, x, t \mid$ sharp valley $)$ is the PDF of $\delta u(x, y, t)$ and $\delta h(x, y, t)$ conditional on the property that there is at least one sharp valley in $[y, y+x)$. Since by definition of number density of sharp valleys $\rho$ we have

$$
\begin{gathered}
p_{n s}=1-\rho|x|+o(x) \\
P^{\delta}(\xi, \omega, x, t \mid \text { sharp valley })=R(\xi, \omega, x, t)+O(1)
\end{gathered}
$$

where $R(\xi, s, x, t)$ is the PDF of $\xi=h\left(y_{0}+x\right)-h\left(y_{0}\right)$, $s\left(y_{0}, t\right)$ and $x$, conditional that $y_{0}$ be a shock position.

$$
\begin{aligned}
& p_{n s}(x, t) P^{\delta}(\xi, \omega, x, t \mid \text { no sharp valley }) \\
& =(1-\rho|x|) \frac{1}{x^{2}} Q\left(\frac{\xi}{x}, \frac{\omega}{x}, t\right)+o(x)
\end{aligned}
$$

here $Q\left(\eta_{h}, \eta_{u}, t\right)$ is the PDF of $\eta_{h}(x, t)$ and $\eta_{u}(x, t)$, the regular part of the velocity and the velocity gradient, respectively. Indeed we have considered the case $x>0$. The case $x<0$ can be treated similarly. We note that, in the limit $x \rightarrow 0$, because of dealing with regular points, we have

$$
x^{2} P^{\delta}\left(x \eta_{h}, x \eta_{u}, x, t\right) \rightarrow Q\left(\eta_{h}, \eta_{u}, t\right)
$$

It implies that

$$
P^{\delta}(\xi, \omega, x, t)=\delta(\omega) \delta(\xi)+o(1)
$$

Define

$$
\begin{aligned}
A(\xi, \omega, t) & =\lim _{x \rightarrow 0} x^{-1}\left(P^{\delta}(\xi, \omega, x, t)-\delta(\omega) \delta(\xi)\right) \\
& =\lim _{x \rightarrow 0} P_{x}^{\delta}(\xi, \omega, x, t) .
\end{aligned}
$$

Taking the limit as $x \rightarrow 0$ in the equation for $P^{\delta}$ ( eq.(23) ) and considering that the system has reached to the stationary state, it follows that $A$ satisfies

$$
\begin{aligned}
& 0=-\alpha \omega A-2 \alpha \int d \omega^{\prime} H\left(\omega,-\omega^{\prime}\right) A\left(\xi, \omega^{\prime}, x, t\right) \\
& (43)+B(\xi, \omega, t)
\end{aligned}
$$

where we have used $\lim _{x \rightarrow 0}(K(0)-K(x))=0$ and also we defined

$$
B(\xi, \omega, t)=\lim _{x \rightarrow 0} G^{\delta}(\xi, \omega, x, t) .
$$

To evaluate $B$ note that as $x \rightarrow 0$

$$
\delta u_{ \pm}\left(x, y_{0}, t\right) \rightarrow 0
$$

This implies that, as $x \rightarrow 0$,

$$
U_{ \pm}(s, \xi, \omega, x, t) \rightarrow S(s, t) \delta(\omega) \delta(\xi)
$$

where $S(s, t)$ is the PDF of $s\left(y_{0}, t\right)$ conditional on $y_{0}$ being a sharp valley location. Hence, from the expression for $G^{\delta}$,

$$
\begin{gathered}
B(\xi, \omega, t)=\alpha \rho \omega S(\omega, t) \delta(\xi)+\alpha \rho<s>\delta(\omega) \delta(\xi) \\
+2 \alpha \rho \delta(\xi) \int_{-\infty}^{\omega} d \omega^{\prime} S\left(\omega^{\prime}, t\right)-2 \alpha \rho H(\omega) \delta(\xi)
\end{gathered}
$$

where $H(\cdot)$ is the Heaviside function and we used $S(s, t)=0$ for $s>0$ since $s\left(y_{0}, t\right) \leq 0$. Inserting this expression in (49), the solution of this equation is

$$
A(\xi, \omega, t)=\left(-\delta(\omega)+\rho\langle s\rangle \delta^{1}(\omega)+\rho S(\omega, t)\right) \delta(\xi)
$$

Here $\delta^{1}(\omega)=d \delta(\omega) / d \omega$ and $\omega$ used the identity $\omega \delta^{1}(\omega)=-\delta(\omega)$. Using the fact that $\rho<s>=-\left\langle\eta_{u}\right\rangle$ [47], we can be restated $A(\xi, \omega, t)$ as

$$
A(\xi, \omega, t)=\left(-\delta(\omega)-\left\langle\eta_{u}\right\rangle \delta^{1}(\omega)+\rho S(\omega, t)\right) \delta(\xi) .
$$

Hence, combining the above results, we have

$$
\begin{aligned}
P^{\delta}(\xi, \omega, x, t) & =\left(\delta(\omega)-x\left(\delta(\omega)+\left\langle\eta_{u}\right\rangle \delta^{1}(\omega)-\rho S(\omega, t)\right)\right) \delta(\xi) \\
& +o(x) .
\end{aligned}
$$

Which is correct for $x>0$. We Reorganize this expression as 
$P^{\delta}(\xi, \omega, x, t)=\left[(1-\rho x)\left(\delta(\omega)-x\left\langle\eta_{u}\right\rangle \delta^{1}(\omega)\right)+x \rho S(\omega, t)\right] \delta(\xi) \quad$ Because the singular part of $Z$ is cancelled by $g$, the first term should be of order of $o(x)$

$$
+o(x)
$$

and then we use the identity

$$
\delta(\omega)-x\left\langle\eta_{u}\right\rangle \delta^{1}(\omega)=\frac{1}{x^{2}} Q\left(\frac{\xi}{x}, \frac{\omega}{x}, t\right)+o(x) .
$$

Now we decompose the fields $h$ and $u$ in terms of the their regular and singular parts as

$$
h_{x}(x, t)=\eta_{h}(x, t)+\sum_{j} \epsilon\left(y_{j}, t\right) \delta\left(y-y_{j}\right)
$$

and

$$
u_{x}(x, t)=\eta_{u}(x, t)+\sum_{j} s\left(y_{j}, t\right) \delta\left(y-y_{j}\right)
$$

So if we let $f^{\delta}(\omega, x, t)$ be defined as

$$
f^{\delta}(\xi, \omega, x, t)=(1-\rho|x|) \frac{1}{x} Q\left(\frac{\xi}{x}, \frac{\omega}{x}, t\right)+|x| \rho R(\xi, \omega, x, t)
$$

then we can write

$$
P^{\delta}(\xi, \omega, x, t)=f^{\delta}(\xi, \omega, x, t)+o(x) .
$$

Now we can prove eq.(25) for $0 \leq b \leq 1$ and an arbitrary value of $a$. The proof for other values of $b$ is similar. Let

$$
\begin{aligned}
& f^{\delta}(\xi, \omega, x, t) \\
= & (1-\rho|x|) \frac{1}{x^{2}} Q\left(\frac{\xi}{x}, \frac{\omega}{x}, t\right)+|x| \rho R(\xi, \omega, x, t), \\
& g^{\delta}(\xi, \omega, x, t)= \\
& {\left[\delta(\omega)-|x|\left(\rho \delta(\omega)+\langle\xi\rangle \delta^{1}(\omega)-\rho S(\omega, t)\right)\right] \delta(\xi) }
\end{aligned}
$$

Because the sharp valley points have contribution in large $\omega$ 's we can write for $M>0$

$$
\begin{aligned}
& \int d \xi d \omega|\xi|^{a}|\omega|^{b}\left(Z^{\delta}-f^{\delta}\right) \\
= & \int_{|\omega| \leq M} d \xi d \omega|\xi|^{a}|\omega|^{b}\left(Z^{\delta}-f^{\delta}\right) \\
+ & \int_{|\omega|>M} d \xi d \omega|\xi|^{a}|\omega|^{b}\left(Z^{\delta}-f^{\delta}\right) .
\end{aligned}
$$

Because of eq.(52), the first term at the rhs of eq.(54) is $o(x)$. To estimate the second term, note that for $M$ large enough

$$
\begin{aligned}
& \int_{|\omega|>M} d \xi d \omega|\xi|^{a}|\omega|^{b} Z^{\delta} \leq \int_{|\omega|>M} d \xi d \omega|\xi|^{a} \omega^{2} Z^{\delta} \\
\leq & \left.\left.\left|\int d \xi d \omega\right| \xi\right|^{a} \omega^{2}\left(Z^{\delta}-g^{\delta}\right)\left|+\int_{|\omega|>M} d \xi d \omega\right| \xi\right|^{a} \omega^{2} g^{\delta} \\
= & o(x)+|x| \rho \int_{|\omega|>M} d \xi d \omega|\xi|^{a} \omega^{2} S(\omega, t) \delta(\xi) \\
= & o(x) .
\end{aligned}
$$

$$
\begin{aligned}
& \int_{|\omega|>M} d \xi d \omega|\xi|^{a}|\omega|^{b} f^{\delta} \\
= & |x|^{a+b}(1-|x| \rho) \int_{\left|\eta_{u}\right|>M / x} d \eta_{h} d \eta_{u}\left|\eta_{h}\right|^{a}\left|\eta_{u}\right|^{b} Q\left(\eta_{h}, \eta_{u}, t\right) \\
+ & |x| \rho \int_{\omega>M} d \xi d \omega|\xi|^{a}|\omega|^{b} R(\xi, \omega, x, t)=o\left(x^{a+b}\right) .
\end{aligned}
$$

We can write $R(\xi, \omega, x, t)$ as $\frac{1}{2} R\left(\xi_{+}, \omega,|x|, t\right)+\frac{1}{2} R\left(\xi_{-}, \omega,-|x|, t\right)$ where for $R\left(\xi_{+}, \omega,|x|, t\right)$ and $R\left(\xi_{-}, \omega,|x|, t\right)$, we have the condition that $\xi=h\left(y_{0}+|x|\right)-h\left(y_{0}\right)$ and $\xi=h\left(y_{0}+|x|\right)-h\left(y_{0}\right)$, respectively. When $x \rightarrow 0$ we can write $\xi_{ \pm}=u_{ \pm}|x|$.

Since $M$ can be made arbitrarily large, we get

$$
\int d \xi d \omega|\xi|^{a}|\omega|^{b}\left(Z^{\delta}-f^{\delta}\right) \leq o\left(x^{a+b}\right)+\delta_{M} O(x)
$$

where $\delta_{M} \rightarrow 0$ as $M \rightarrow+\infty$. Noting that

$$
\begin{aligned}
& \int d \xi d \omega|\xi|^{a}|\omega|^{b} f^{\delta} \\
= & \begin{cases}|x|^{a+b}\left\langle\left|\eta_{h}\right|^{a}\left|\eta_{u}\right|^{b}\right\rangle & \text { if } 0 \leq b<1 \\
|x|^{a+1}\left(\left\langle\left|\eta_{h}\right|^{a}\left|\eta_{u}\right|\right\rangle+\frac{1}{2} \rho\left\langle|s|\left(\left|u_{+}\right|^{a}+\left|u_{-}\right|^{a}\right)\right\rangle\right) & \text { if } b=1\end{cases}
\end{aligned}
$$

We obtain (25) for $0 \leq b \leq 1$. For $b>1$ the leading term in our calculation, will be the second term of the eq.(56) with the order of $o\left(x^{a+1}\right)$. The leading term is $\frac{1}{2} \rho\left\langle|s|\left(\left|u_{+}\right|^{a}+\left|u_{-}\right|^{a}\right)\right\rangle$.

Also there is an alternative method to prove the eq.(25) for the case $b>1$. The method is based on the calculation of the mixed moment $\left\langle\left.\left(\mid h\left(x_{1}\right)-h\left(x_{2}\right)\right)\right|^{a}\right|\left(u\left(x_{1}\right)-\right.$ $\left.\left.u\left(x_{2}\right)\right)\left.\right|^{b}\right\rangle$ for integer orders while $b \geq 1$, directly from the $\mathrm{PDF}^{\prime}$ 's equation (23) by integrating over two $\omega$ 's, i.e.

$$
\begin{aligned}
P_{t}^{\delta}= & -\alpha \omega P_{x}^{\delta}-2 \alpha \int d \omega^{\prime} H\left(\omega^{\prime}-\omega\right) P_{x}^{\delta}\left(\xi, \omega^{\prime}, x, t\right) \\
& +2\left(K_{x x}(0)-K_{x x}(x)\right) P_{\omega \omega}^{\delta}+2(K(0)-K(x)) P_{\xi \xi}^{\delta} \\
+ & G^{\delta}(\xi, \omega, x, t) .
\end{aligned}
$$

In the limit $x \rightarrow 0$, keeping $\sigma$ finite, and in stationary state, it will be simplified to

$$
\begin{aligned}
0 & =-\alpha \omega P_{x}^{\delta}-2 \alpha \int d \omega^{\prime} H\left(\omega^{\prime}-\omega\right) P_{x}^{\delta}\left(\xi, \omega^{\prime}, x, t\right) \\
& +G^{\delta}(\xi, \omega, x, t)
\end{aligned}
$$

First of all the term $\int d \xi d \omega|\xi|^{n}|\omega|^{m} G^{\delta}(\xi, \omega, x, t)$ should be calculated in the $x \rightarrow \pm 0$ limit. This can be done by using the relation (43). Note that

$$
\int d \xi d \omega|\xi|^{n}|\omega|^{m} G^{\delta}(\xi, \omega, x, t)=
$$




$$
\begin{aligned}
& \alpha \frac{\rho}{2}\left\langle s\left|\left(\delta u_{+}+\operatorname{sgn}(x) s\right)\right|^{m}\left|\left(\delta h_{+}+\operatorname{sgn}(x) \epsilon\right)\right|^{n}\right\rangle \\
+ & \alpha \frac{\rho}{2}\left\langle s\left|\delta u_{+}\right|^{n}\left|\delta h_{+}\right|^{n}\right\rangle \\
- & \alpha \rho \int_{0}^{1} d \beta\left\langle s\left|\left(\delta u_{+}+\beta \operatorname{sgn}(x) s\right)\right|^{m}\left|\left(\delta h_{+}+\operatorname{sgn}(x) \frac{\epsilon}{2}\right)\right|^{n}\right\rangle \\
+ & \alpha \frac{\rho}{2}\left\langle s\left|\left(\delta u_{-}+\operatorname{sgn}(x) s\right)\right|^{m}\left|\left(\delta h_{-}+\operatorname{sgn}(x) \epsilon\right)\right|^{n}\right\rangle \\
+ & \alpha \frac{\rho}{2}\left\langle s\left|\delta u_{-}\right|^{m}\left|\delta h_{-}\right|^{n}\right\rangle \\
- & \alpha \rho \int_{0}^{1} d \beta\left\langle s\left|\left(\delta u_{-}+\beta \operatorname{sgn}(x) s\right)\right|^{m}\left|\left(\delta h_{-}+\operatorname{sgn}(x) \frac{\epsilon}{2}\right)\right|^{n}\right\rangle .
\end{aligned}
$$

If we go back and look carefully to the definition $\delta u_{ \pm}$, we see that $\delta h_{ \pm} \simeq u_{ \pm}|x| \simeq o(x)$ and $\delta u_{ \pm} \simeq o(x)$ as $x \rightarrow 0$. While it should be realized that the sharp valley strength $s$ is of the order $O(1)$ as $\epsilon \rightarrow 0$, so in the limit $x \rightarrow 0$, the result of the integral would be simplified as

$$
\begin{aligned}
& \int d \xi d \omega|\xi|^{n}|\omega|^{m} G^{\delta}(\xi, \omega, x, t) \simeq \\
& \frac{\alpha}{2}\left(\frac{m-1}{m+1}\right) \rho|x|^{n+1}\left\langle|s|^{m+1}\left(\left|u_{+}\right|^{n}+\left|u_{-}\right|^{n}\right)\right\rangle .
\end{aligned}
$$

Finally multiplying the terms of equation (57) in $|\xi|^{n}$ and $|\omega|^{m}$ and integrating respect to $\xi$ and $\omega$ we have

$$
\begin{aligned}
& \left\langle|\xi|^{n}|\omega|^{m+1}\right\rangle= \\
& \frac{1}{2} \rho|x|^{n+1}\left\langle|s|^{m+1}\left(\left|u_{+}\right|^{n}+\left|u_{-}\right|^{n}\right)\right\rangle \quad \text { if } \quad m \geq n \in \mathcal{N}
\end{aligned}
$$

where the result coincides perfectly with eq.(25) which is the general form of eq.(60).

[1] A.-L. Barabasi and H.E. Stanley, Fractal Concepts in Surface Growth, (Cambridge University Press, New York,1995).

[2] T. Halpin-Healy and Y.C.Zhang, Phys.Rep. 254,218(1995); J. Krug, Adv. Phys. 46, 139 (1997).

[3] J. Krug and H. Spohn "In solids far from equilibrium growth, Morphology and defects", edited by C.Godreche(Cambridge University Press,New York,1990).

[4] P. Meakin Fractals,Scaling and Growth Far from Equilibrium( Cambridge University Press, Cambridge ,1998).

[5] Mehran Kardar, Physica A 281, 295 (2000).

[6] M. Kardar, G. Parisi and Y.C. Zhang, Phys. Rev. Lett. 56, 889 (1986).

[7] L.Golubovic and Z.G.Wang, Phys.Rev.E 49,2567(1994)

[8] I. Ispolatov, P.L. Krapirsky and S. Redner, Phys.Rev.E 52, 2540 (1995).
[9] J. Krug and H. Spohn, Europhys Lett.8, 219 (1989).

[10] $\mathrm{H}$. Van Beijeren, R. Kutner and H. Spohn, Phys.Rev.Lett.54, 2026 (1985).

[11] H.k. Janssen and B. Schmittmann, Z. Phys.B. 63, 517 (1986).

[12] G. Blatter, M.V. Feigelman, V.B. Geshkenbein, A.I. Larkin and V.M. Vinokur, Rev. Mod. Phys. 66,1125 (1994).

[13] D.A. Huse,C.l. Henley and D.S. Fisher,Phys.Rev.Lett.55, 2924(1985).

[14] M. Kardar and Y.C.Zhang,Phys.Rev.Lett.58,2087(1987).

[15] D.S. Fisher and D.A. Huse, Phys.Rev.B43,10728(1991).

(59) [16] R.D. Kamien, P.Le. Doussal and D.R. Nelson, Phys. Rev. A.45,8727 (1992).

[17] H.C. Fogedby, A.B. Ericksson and L.V. Mikheev, Phys. Rev. Lett. 75,1883 (1995).

[18] L. Balents, J-P. Bouchaud and M. Mezard, condmat/9601137.

[19] R. Bundschuh and M. Lassig, cond-mat/9602045.

[20] G. Parisi and F. Slanina, cond-mat/9712208.

[21] D.A. Gorokhov and G. Blatter, Phys. Rev. lett. 82,2705 (1999).

[22] P. Delos Rios, Phys. Rev. Lett. 82,4236 (1999).

[23] A. Basu, Phys. Rev. E62,4675 (2000).

[24] E. Brunet and B. Derrida, Phys. Rev. E 61,6789 (2000).

[25] E. Perlsman and S. Havlin, Phys. Rev. E63,010102(R) (2001).

[26] R. Mohayaee, A.L. stella and C. Vander Zande, condmat/0101091.

[27] M.V. Feigelman, Sov. Phys. JETP. 52,555(1980)[Zh. Eksp. Teor. Fiz. 79,1095(1980)].

[28] S.F. Shandarin,Ya.B. Zeldovich, Rev. Mod. Phys. 61,185 (1989).

[29] M. Vergassola, B. Dubrulle, U. Frisch and A. Noullez, Astron. Astrophys, 280, 325 (1994).

[30] S.F. Shandarin, astro-phy/9507082.

[31] U. Frisch, J. Bec and B. Villone, cond-mat/9912110.

[32] J. P. Bouchaud, M. Mezard and G.Parisi, Phys. Rev. E 52, 5116 (1995).

[33] M. Mezard, cond-mat/9801029.

[34] H. C. Fogedby, Phys. Rev. E 57, 2331 (1998); Phys. Rev. Lett. 80, 1126 (1998); Phys. Rev. E 57, 4993 (1998); Phys. Rev. E 59, 5065 (1999); Phys. Rev. E 66, 4950 (1999).

[35] V. Yakhot and A. Chekhlov, Phys. Rev. Lett. 77, 3118 (1996).

[36] A.Chekhlov and V. Yakhot, Phys.Rev.E 51, R2739 (1995).

[37] A. Polyakov, Phys. Rev. E 52, 6183 (1995).

[38] E. Balkovsky, G. Falkovich, I. Kolokolov and V. Lebedev, JETP. Lett. 61, 1012 (1995); Phys. Rev. Lett. 78, 1452 (1997).

[39] G. Falkovich and V. Lebedev,e-print chao-dyn/9708002

[40] V. Gurarie and A. Migdal, Phys. Rev. E 54, 4908 (1996).

[41] J. P. Bouchaud and M. Mezard Phys. Rev. E 54, 5116 (1996).

[42] T. Gotoh and R. H. Kraichnan, chao-dyn/9803037.

[43] S. Boldyrev, Phys. Rev. E 55, 6907 (1997).

[44] S. Boldyrev, hep-th/9707255; hep-th/9805100. 
[45] V. Yakhot, Phys. Rev. E 57, 1737 (1997). chaodyn/9904016; chao-dyn/9909017; chao-dyn/0001027.

[46] W.E,

K.Khanin, A.Mazel and Ya.G.Sinai, Phys.Rev.Lett. 78 , 1904 (1997).

[47] W. E and E. Vanden Eijnden, Phys.Rev.Lett.83,2572(1999) ; chao-dyn/9901006; chao-dyn/9904028; chao-dyn/9901029.

[48] R.H. Kraichnan, Phys. Fluids 11,3738 (1999).

[49] T. Gotoh and R.H. Kraichnan, Phys. Fluids A 5,445 (1993) ; Phys. Fluids 10,2859 (1998).

[50] J. Bec and U. Frisch, cond-mat/9906047; J. Bec, nlin.CD/0103029.

[51] J. Bec, U. Frisch, K. Khanin, chao-dyn/9910001.

[52] D. Bernard and K. Gawedzki, chao-dyn/9805002.

[53] M. Bauer and D. Bernard, chao-dyn/9812018.

[54] L. Frachebourg and Ph. A. Martin, cond-mat/9905056.

[55] S. N. Gurbatov, chao-dyn/9912011.

[56] F. Hayot and C. Jayaprakash, nlin.CD/0005050; chaodyn/9901026.

[57] U. Frisch, J. Bec, nlin.CD/0012033,proceedings Les Houches 2000 "New Trends in Turbulence".

[58] D. Bernard, cond-mat/0007106.

[59] J. Davoudi, A.A. Masoudi, M.R. Rahimi Tabar, A.R. Rastegar and F. Shahbazi,Phys. Rev. E 63, 056308 (2001).

[60] H.C. Fogedby,cond-mat/0201168.

[61] L.H. Tang and H. Leschorn, Phys. Rev. A45, 7162 (1992)

[62] T. Ala-Nissila, T. Hielt, J.M. Kosterlitz and O. Venalainen, J. Stat. Phys. 72, 207 (1993).

[63] J.M. Kim, Phys. Rev. lett 80, 888 (1998).

[64] C.S. Chin and M. den Nijs, cond-mat/9810083.

[65] M. Prahofer and H. Spohn, cond-mat/9912264.

[66] M.K. Verma, Physica A277,359 (2000).

[67] E. Marinari, A. Pagnani and G. Parisi, J. Phys. A: Math. Gen. 33,8181 (2000).

[68] M.A. Moore et al, Phys.Rev.Lett. 74, 4257 (1995).

[69] T.J. Newman and H. Kallabis, cond-mat/9512104.

[70] T.J. Newman and A.J. Bray, cond-mat/9604071.

[71] S. Stepanow, Phys. Rev. E 55, R4853 (1997).

[72] M. Lassig, Nucl. Phys. B448 (1998).

[73] C. Castellano, M. Marsili and L. Pietronero, Phys. Rev. Lett. 80,3525 (1998) .

[74] C. Castellano, A.Gabrielli, M. Marsili, M.A Munoz and L. Pietronero, Phys. Rev. E58,R5209 (1998).

[75] C. Castellano, M. Marsili, M.A. Munoz and L. Pietronero, cond-mat/9904434.

[76] H.K. Janssen, U.C. Taeuber and E. Frey, Eur. Phys. J. B 9 ,491(1999).

[77] A. A. Masoudi, F. Shahbazi, J. Davoudi and M. Reza Rahimi Tabar, Phys. Rev. E 65, 026132(2002).

[78] R.Peyret, Computational Fluid Mechanics(Academic Press, 2000).

[79] R.W. Hockney and J.W. Eastwood, Computer Simulation Using Particles( Institute of Physics Publishing, 1992).

[80] T.J. Newman and A.J. McKane, Phys. Rev. E 55, 165175 (1997).

[81] J.M.Kim, M.A.Moore, and A.J.Bray,Phys.Rev.A /big 44,2345(1991. 